\title{
Branching random walks in random environment on Cayley graphs
}

\section{Serguei Popov}

Instituto de Matemática e Estatística, Universidade de São Paulo, rua do Matão 1010, CEP 05508-090, São Paulo SP, Brasil

E-mail address: popov@ime.usp.br

$U R L$ : http://www.ime.usp.br/ popov

\begin{abstract}
We review some recent results concerning recurrence and transience for branching random walks in random environment on $d$ dimensional lattices and on trees. We obtain some generalizations of these results for the case when the branching random walk in random environment takes place on arbitrary infinite connected Cayley graph.
\end{abstract}

\section{Introduction}

In this paper we are interested mainly in studying transience/recurrence of general branching random walks in random environment on Cayley graphs. Roughly speaking, the model is the following. Start with one particle at the origin of the graph. Then, at every (discrete) moment each particle produces a random configuration of offspring in the set of neighbouring sites, independently of other particles. This random configuration is generated according to a certain measure, which depends on the particle's location but does not depend on time. These measures themselves are supposed to be random, and, before the branching random walk starts, they are placed into the sites of the graph in an i.i.d. way. We study a fairly general case, i.e., the branching and transition mechanisms are not supposed to be independent, and, moreover, the immediate offspring of a particle should not be necessarily independent either (we allow, for instance, the following situation: if a particle has two children, then necessarily they jump to the same place). We suppose also that particles always generate at least one offspring, so that the global extinction is not possible.

2000 Mathematics Subject Classification. 60K37, 60J10, 60J80.

Key words: recurrence, transience, branching random walk. 
Branching random walks in random environment are useful as microscopic models for reaction-diffusion-convection phenomena in a space inhomogeneous medium. This model received considerable attention in the past few years. A multidimensional $(d \geq 3)$ branching random walk for which the transition probabilities are those of the simple random walk, and the particles can branch only in some special sites (randomly placed, with a decreasing density) was considered by den Hollander, Menshikov and Popov [10], and several sufficient conditions for recurrence and transience were obtained. A modification of that model was considered by Volkov in [15]. Branching random walks in many-dimensional random environment are considered also in $[1,14]$, where some questions related to the local and total particle populations are studied.

Dimension $d=1$ leads to more explicit results, thanks to the order structure (see, e.g., $[3,11])$. In the case $d=1$ with nearest neighbor jumps, particles have to visit all intermediate locations, and, for a locationindependent jump law, Greven and den Hollander [9] and Baillon, Clément, Greven and den Hollander [2] could prove some useful variational formulas. As can be seen in [12], the case where particles move on the tree has a flavour similar to $d=1$. The case of inhomogeneous jumps with constant branching rate can be formulated as a tree-indexed random walk. In this case, a complete classification of recurrence/transience is obtained by Gantert and Müller [8], involving the branching rate and the spectral radius of the transition operator. In [13] this classification is obtained for the case when the branching and transition environments are chosen independently from each other. Some results concerning the speed of the rightmost particle for the case when the extinction is possible were obtained by Devulder in $[5]$.

In this paper we are mainly interested in the transience and recurrence properties of the model. As mentioned above, in the case of constant branching or when the branching and the transitions are independent, this problem is well understood. When the branching and the transitions are not independent, some progress was achieved in $[3,11,12,4]$, and here we summarize and provide some generalizations of the results of those papers.

\section{Formal definitions and basic properties of the model}

Suppose that $\mathcal{G}$ is a finitely generated infinite group. We usually write the group operation multiplicatively (except for the case $\mathcal{G}=\mathbb{Z}^{d}$ ), and $\mathbf{e}$ stands for the neutral element. For a fixed set $S \subset \mathcal{G}$, the Cayley graph $\mathfrak{G}(\mathcal{G}, S)$ is defined as a graph with the vertex set $\mathcal{G}$, and the edge set $\mathcal{E}(\mathcal{G}, S)$, where

$$
\mathcal{E}(\mathcal{G}, S)=\left\{(x, y): x^{-1} y \in S\right\}
$$


(equivalently, from $x$ the edges go to $x s, s \in S$ ). Throughout this paper we suppose that $S$ is a generating set of $\mathcal{G}$, so that the graph is connected. Note that, in general, we do not suppose that $S$ is symmetric, so the graph can be oriented. We refer to the site corresponding to e as the origin of the graph.

Now, let us describe the model. Define (with $\mathbb{Z}_{+}=\{0,1,2, \ldots\}$ )

$$
\mathcal{V}=\left\{v=\left(v_{s}, s \in S\right): v_{s} \in \mathbb{Z}_{+}, \sum_{s \in S} v_{s} \geq 1\right\},
$$

and for $v \in \mathcal{V}$ put $|v|=\sum_{s \in S} v_{s}$; note that $|v| \geq 1$ for all $v \in \mathcal{V}$. Furthermore, define $\mathcal{M}$ to be the set of all probability measures $\omega$ on $\mathcal{V}$ :

$$
\mathcal{M}=\left\{\omega=(\omega(v), v \in \mathcal{V}): \omega(v) \geq 0 \text { for all } v \in \mathcal{V}, \sum_{v \in \mathcal{V}} \omega(v)=1\right\} .
$$

Finally, let $Q$ be a probability measure on $\mathcal{M}$. Now, for each $x \in \mathcal{G}$ we choose a random element $\omega_{x} \in \mathcal{M}$ according to the measure $Q$, independently. The collection $\boldsymbol{\omega}=\left(\omega_{x}, x \in \mathcal{G}\right)$ is called the environment. Given the environment $\boldsymbol{\omega}$, the evolution of the process is described in the following way: start with one particle at some fixed site of $\mathcal{G}$. At each integer moment the particles branch independently using the following mechanism: for a particle at site $x \in \mathcal{G}$, a random element $v=\left(v_{s}, s \in S\right)$ is chosen with probability $\omega_{x}(v)$, and then the particle is substituted by $v_{s}$ particles in $x s$ for all $s \in S$.

We denote by $\mathbb{P}, \mathbb{E}$ the probability and expectation with respect to $\boldsymbol{\omega}$ (in fact, since the environment is i.i.d., $\mathbb{P}=\bigotimes_{x \in \mathcal{G}} Q_{x}$, where $Q_{x}$ are copies of $Q$ ), and by $\mathrm{P}_{\omega}^{x}, \mathrm{E}_{\omega}^{x}$ the (so-called "quenched") probability and expectation for the process starting from $x$ in the fixed environment $\boldsymbol{\omega}$.

As already mentioned, this notion of branching random walk is more general than that of $[3,11,12]$, since here we do not suppose that the immediate descendants of a particle jump independently.

Suppose that the two conditions below are fulfilled:

\section{Condition B.}

$Q\{\omega:$ there exists $v \in \mathcal{V}$ such that $\omega(v)>0$ and $|v| \geq 2\}>0$.

Condition E. There is a set $S^{\prime} \subset S$ which is a generating set for the group $\mathcal{G}$ and such that

$$
Q\left\{\omega: \sum_{v: v_{s} \geq 1} \omega(v)>0 \text { for any } s \in S^{\prime}\right\}=1 .
$$


Condition $\mathrm{B}$ ensures that the model cannot be reduced to random walk without branching, and Condition $\mathrm{E}$ is a natural ellipticity condition which ensures that the walk cannot be reduced to a proper subgroup of $\mathcal{G}$.

Due to Condition B, for almost all environments the population size tends to infinity, as can be seen from Lemma 2.5 below. This shows that the branching random walk is always transient as a process on $\mathbb{Z}_{+}^{\mathcal{G}}$. So, we introduce more appropriate notions of recurrence and transience.

Definition 2.1. For the particular realization of the random environment $\boldsymbol{\omega}$, the branching random walk is called recurrent if

$$
\mathrm{P}_{\omega}^{0}[\text { the origin is visited infinitely often }]=1 .
$$

Otherwise, the branching random walk is called transient.

By the Markov property, the recurrence is equivalent to

$$
\mathrm{P}_{\omega}^{0}[\text { the origin is visited at least once }]=1 .
$$

In principle, the above definition could depend on the starting point of the process and on the realization of the environment $\boldsymbol{\omega}$ (in fact, as Example 1 below shows, this can be the case when the environment is nonrandom). It is possible to prove, however, that a natural dichotomy takes place:

Proposition 2.2. We have either:

(i) For $\mathbb{P}$-almost all $\boldsymbol{\omega}$, the branching random walk is recurrent, in which case $\mathrm{P}_{\omega}^{x}[$ the origin is visited infinitely often $]=1$ for all $x \in$ $\mathcal{G}$, or:

(ii) For $\mathbb{P}$-almost all $\boldsymbol{\omega}$, the branching random walk is transient, in which case $\mathrm{P}_{\omega}^{x}[$ the origin is visited infinitely often $]=0$ for all $x \in$ $\mathcal{G}$.

It is not difficult to construct (see e.g. the example after the proof of Theorem 4.3 in [3]) environments $\boldsymbol{\omega}$ such that $\mathrm{P}_{\omega}^{x}$ [0 is visited infinitely often] is strictly between 0 and 1. The next example (which is Example 1 from [4]) shows that randomness of the environment is essential for our statements (and also shows, by the way, that there is no hope to prove Proposition 2.2 by arguments of the type "recurrence should not be sensitive to changes of the environment in finite regions").

Example 1. Let $\mathcal{G}=\mathbb{Z}, S=\{-1,1\}$, and consider two measures $\omega^{(1)}, \omega^{(2)}$ :

(i) under $\omega^{(1)}$, with probability $2 / 3$ there is only one child which is located one step to the left and with probability $1 / 3$ there is only one child which is located one step to the right; 
(ii) under $\omega^{(2)}$, with probability $1 / 3$ there is only one child which is located one step to the right and with probability $2 / 3$ there are two children one being located to the right and the other to the left.

If all sites $x<0$ have the environment $\omega^{(1)}$ (we say they are of type 1 ) and all sites $x \geq 0$ are of type 2 , we have $\mathrm{P}_{\omega}^{x}[0$ is visited infinitely often] is 1 for $x \geq 0$ and is less than 1 for $x<0$. Changing the site $x=0$ from type 2 to type 1 turns the branching random walk from recurrent to transient. This example also shows that, in general, the recurrence does depend on the environment locally. Moreover, it shows that $\mathrm{P}_{\omega}^{0}[$ the origin is visited infinitely often] may be different from 0 and 1 . We will see below that, selecting randomly the environment in an i.i.d. fashion, makes this branching random walk recurrent (for this particular example it follows e.g. from Theorem 1.5 of [4]).

Similarly to $[3,4,8,11,12,13]$, for this model it holds that transience and recurrence of the process only depend on the support of the measure $Q$, i.e., the smallest closed subset $F \subset \mathcal{M}$ such that $Q(F)=1$. Note that $\omega$ belongs to the support if and only if $Q(\mathcal{N})>0$ for all neighborhood $\mathcal{N}$ of $\omega$ in $\mathcal{M}$.

Theorem 2.3. Suppose that the branching random walk is recurrent (respectively, transient) for almost all realizations of the random environment from the distribution $Q$. Then for any measure $Q^{\prime}$ (which satisfies Condition $E)$ with $\operatorname{supp} Q \subseteq \operatorname{supp} Q^{\prime}\left(\right.$ respectively, $\left.\operatorname{supp} Q^{\prime} \subseteq \operatorname{supp} Q\right)$ the process is recurrent (respectively, transient) for almost all realizations of the random environment from the distribution $Q^{\prime}$.

The fact that recurrence and transience only depend on the support of the measure $Q$ is quite natural for this kind of model. Besides $[3,4,8,11$, $12,13]$, we can mention also [6]: in Theorem 3 of that paper it is shown that, for the branching diffusion, the intensity of 'mild' Poissonian obstacles plays no role for exponential growth and local extinction.

\subsection{Seeds}

To prove Proposition 2.2 and Theorem 2.3, we have to introduce the notion of a seed:

Definition 2.4. Fix a finite set $U \subset \mathcal{G}$ such that $\mathbf{e} \in U$, and $H_{x} \subset \mathcal{M}$ with $Q\left(H_{x}\right)>0$ for all $x \in U$. With $H=\left(H_{x}, x \in U\right)$, the couple $(U, H)$ is called a seed. We say that $\boldsymbol{\omega}$ has a $(U, H)$-seed at $z \in \mathcal{G}$ if

$$
\omega_{z x} \in H_{x} \text { for all } x \in U
$$

and that $\boldsymbol{\omega}$ has a $(U, H)$-seed in the case $z=\mathbf{e}$. We call $z$ the center of the seed. 
Lemma 2.5. With probability 1 the branching random walk visits infinitely many distinct $(U, H)$-seeds (to visit the seed means to visit the site where the seed is centered).

Proof. The idea of the proof is the following: since the environment is i.i.d., we are not obliged to construct it completely in the beginning, but we can rather reveal it gradually, as new sites get visited by the particles. In particular, the following situation will happen infinitely often: at a given moment (say, $t$ ) a site (say, $x$ ) is visited for the first time, and there exists $y \in U$ such that all the sites of the set $x y^{-1} U=\left\{x y^{-1} u, u \in U\right\}$ were unvisited by time $t-1$. This means that $x$ may be a part of an $(U, H)$-seed centered in $x y^{-1}$. Using the Borel-Cantelli lemma and Condition E, it is elementary to show that infinitely many seed centers will be visited.

As we will see, the notion of seed becomes powerful when combined with independence of the medium. Hence we give two more definitions (cf. Definitions 2.4 and 2.5 of [4]).

Definition 2.6. For a particular realization of the random environment $\boldsymbol{\omega}$, we define the branching random walk restricted on set $M \subset \mathbb{Z}^{d}$ simply by discarding all particles that step outside $M$, and write $\mathrm{P}_{\omega \mid M}, \mathrm{E}_{\omega \mid M}$ for corresponding probability and expectation.

The next definition is a particular case of Definition 2.5 of [4].

Definition 2.7. Let $U$ be a finite subset of $\mathcal{G}$ with $\mathbf{e} \in U$. Let $\mathbf{p}$ be a probability distribution on $\mathbb{Z}_{+}$with mean larger than 1 , i.e., $\mathbf{p}=\left(p_{0}, p_{1}, p_{2}, \ldots\right)$ with $p_{i} \geq 0, \sum p_{i}=1, \sum i p_{i}>1$. An $(U, H)$-seed is called $\mathbf{p}$-recurrent if for any $\boldsymbol{\omega}$ such that $\omega_{x} \in H_{x}, x \in U$, we have

$$
\mathrm{P}_{\omega \mid U}^{y}[\mathbf{e} \text { will be visited by at least } i \text { "free" particles }] \geq \sum_{j=i}^{\infty} p_{j}
$$

for all $i \geq 1$ and all $y \in W$. By "free" particles we mean that none is the descendant of another one.

Note that, by definition of the restricted branching random walk, the above probability depends on the environment inside $U$ only.

The next lemma shows the importance of $\mathbf{p}$-recurrent seeds.

Lemma 2.8. Suppose that there exists an $(U, H)$-seed that is $\mathbf{p}$-recurrent for some $\mathbf{p}$. Then this implies the recurrence of the branching random walk for a.e. environment $\boldsymbol{\omega}$.

Proof. The proof of this lemma is quite analogous to that of Lemma 2.6 of [4], so we give only a sketch. By Lemma 2.5, an infinite number of 
$(U, H)$-seeds will be visited almost surely. Now, each time a p-recurrent seed is visited, one can define a Galton-Watson branching process there (by restricting the particles on the set $z U$, where $z$ is the center of the seed; the "descendants" of a particle in $z$ are free particles visiting $z$, see the proof of Lemma 2.6 of [4] for more details). This branching process is supercritical (since the distribution of the first generation dominates $\mathbf{p}$ ), and so it does not get extinct with a positive probability. Moreover, one can consider an infinite number of visited p-recurrent seeds that are nonoverlapping, and so the nonextinctions occur independently. Thus, at least one of those branching processes survives forever, and from this it is straightforward to obtain that the origin is visited infinitely often.

Proof of Proposition 2.2. Again, this is very similar to the proof of Propositions 1.2 and 1.3 from [4], so we give only a sketch. First, if we assume that the event $\left\{\mathrm{P}_{\omega}^{0}[\right.$ the origin is visited infinitely often $\left.]=1\right\}$ has positive $\mathbb{P}$-probability, this would imply that (since the branching is present)

$$
\mathrm{P}_{\omega}^{0}[\text { the origin is visited by (at least) two free particles }]=1 \text {. }
$$

Then, we can take $t$ large enough so that

$$
\mathrm{P}_{\omega}^{0}[\text { the origin is visited by (at least) two free particles before time } t]>3 / 4 \text {. }
$$

Since the jumps are bounded, this probability depends only on a finite piece of the environment, and so we can construct a p-recurrent seed with $\mathbf{p}=(1 / 4,0,3 / 4,0,0, \ldots)$. From Lemma 2.8 , we conclude that the branching random walk is recurrent for $Q$-a.e. environment. Therefore the set of recurrent environments has $\mathbb{P}$-probability 0 or 1 .

On the other hand, using ellipticity one obtains that

$$
\boldsymbol{\omega} \text { recurrent } \Longleftrightarrow \mathrm{P}_{\omega}^{0}[x \text { is visited infinitely often }]=1,
$$

for all $x \in \mathcal{G}$. Since the law of $\boldsymbol{\omega}$ is stationary, this means that the recurrence is also equivalent to

$$
\mathrm{P}_{\omega}^{x}[0 \text { is visited infinitely often }]=1
$$

for all $x \in \mathcal{G}$.

Analogously, assume that with positive $\mathbb{P}$-probability,

$$
\left.\mathrm{P}_{\omega}^{x} \text { [the origin is visited infinitely often }\right]>0
$$

for some $x \in \mathcal{G}$. Similarly to the previous argument, here also it is possible to prove that this implies the existence of a recurrent seed (with another $\mathbf{p}$, see the proof of Proposition 1.3 from [4] for more details), so it remains only to apply Lemma 2.8 once again.

Proof of Theorem 2.3. From Lemma 2.8 and the proof of Proposition 2.2 it can be extracted that the process is recurrent if and only if there exists 
a p-recurrent seed which has a positive $Q$-probability. So, if a measure $Q^{\prime}$ has a bigger support, then that seed has also a positive $Q^{\prime}$-probability, so the process in the environment constructed using $Q^{\prime}$ is recurrent as well. This proves Theorem 2.3.

\section{Conditions for transience and recurrence}

To formulate the sufficient (and sometimes necessary) condition for the transience, we need some definitions.

Definition 3.1. A function $\varphi: \mathcal{G} \rightarrow \mathbb{R} \backslash\{0\}$ is called multiplicative, if $\varphi(x y)=\varphi(x) \varphi(y)$ for all $x, y \in \mathcal{G}$.

Note that, if $\varphi$ is multiplicative, then $\varphi(\mathbf{e})=1$ and $\varphi\left(x^{-1}\right)=(\varphi(x))^{-1}$ for all $x \in \mathcal{G}$.

Definition 3.2. A function $\psi: \mathcal{G} \rightarrow \mathbb{R} \backslash\{0\}$ is called almost multiplicative, if $\psi(\mathbf{e})=1$ and there exist $N, r_{k}(s), k=1, \ldots, N, s \in S$ and nonintersecting sets $G_{k}, k=1, \ldots, N$, such that

$$
\mathcal{G} \backslash\{\mathbf{e}\}=G_{1} \cup \ldots \cup G_{N},
$$

and for all $k=1, \ldots, N, s \in S, x \in G_{k}$ we have

$$
\psi(x s)=r_{k}(s) \psi(x) .
$$

Let us define for $\omega \in \mathcal{M}, s \in S$

$$
\mu_{s}^{\omega}=\sum_{v \in \mathcal{V}} v_{s} \omega(v)
$$

i.e., $\mu_{s}^{\omega}$ is the mean number of particles sent from $x$ to $x s$ when the environment at $x$ is $\omega$.

The next theorem gives a sufficient condition for the transience.

Theorem 3.3. Suppose that there exists a positive function $\psi(x)$ such that there exists $u \in \mathcal{G}$ with $\psi(u)<1, \psi$ is almost multiplicative in the sense of Definition 3.2, and for any $k$ and any $x \in G_{k}$

$$
\sum_{s \in S} \mu_{s}^{\omega} r_{k}(s) \leq 1
$$

for all $\omega \in \operatorname{supp} Q$. Then the branching random walk is transient.

An immediate consequence of this result is the following 
Corollary 3.4. Suppose that on $\mathcal{G}$ there exists a multiplicative function $\varphi$, which is positive and nontrivial (i.e., $\varphi \not \equiv 1$ ), and such that

$$
\sum_{s \in S} \mu_{s}^{\omega} \varphi(s) \leq 1
$$

Then the branching random walk is transient.

(Note that, if $\varphi$ is nontrivial, then there exists $x \in \mathcal{G}$ such that $\varphi(x)<1$.)

Proof of Theorem 3.3. Let $\eta_{n}(y)$ be the number of particles in site $y$ at time $n$. Define

$$
F_{n}=\sum_{y \in \mathcal{G}} \eta_{n}(y) \psi(y) .
$$

Suppose that the process starts from $u \in \mathcal{G}$ with $\psi(u)<1$, and let us also modify the environment in such a way that any particle which enters the origin neither moves nor branches anymore. Using (1), we prove now that the process $\left(F_{n}, n=0,1,2, \ldots\right)$ is a supermartingale:

$$
\begin{aligned}
& \mathrm{E}_{\omega}^{u}\left(F_{n+1} \mid \eta_{1}(\cdot), \ldots, \eta_{n}(\cdot)\right)= \\
& \quad=\eta_{n}(\mathbf{e})+\sum_{k=1}^{N}\left(\sum_{x \in G_{k}} \eta_{n}(x) \psi(x) \times \sum_{s \in S} \mu_{s}^{\omega_{x}} r_{k}(s)\right) \leq F_{n} .
\end{aligned}
$$

Since the process $F_{n}$ is also nonnegative, it converges a.s. as $n \rightarrow \infty$ to some random variable $F_{\infty}$. By Fatou's lemma,

$$
\mathrm{E}_{\omega}^{u} F_{\infty} \leq \mathrm{E}_{\omega}^{u} F_{0}=\psi(u)<1 .
$$

On the other hand, any particle stuck in e contributes at least one unit to $F$. From this we obtain that with positive probability the branching random walk will not enter to the origin, so the proof of Theorem 3.3 is finished.

Concerning the conditions for the recurrence, we can mention that (as shown in the previous section), if there exists a p-recurrent seed, then the branching random walk is recurrent. In many examples this seed can be constructed explicitly; most of the sufficient conditions for the recurrence are proven by showing that, under certain circumstances, it is possible to construct a recurrent seed. See e.g. equations (4), (5), and Theorem 1.5 of [4]. However, in some situations it is possible to obtain necessary and sufficient conditions for transience/recurrence. In fact, what stated in Theorem 3.3, maybe, is also necessary for the transience (and sometimes it is possible to prove that, see Sections 3.1 and 3.2 below); at least, the author does not know of any counterexamples. 


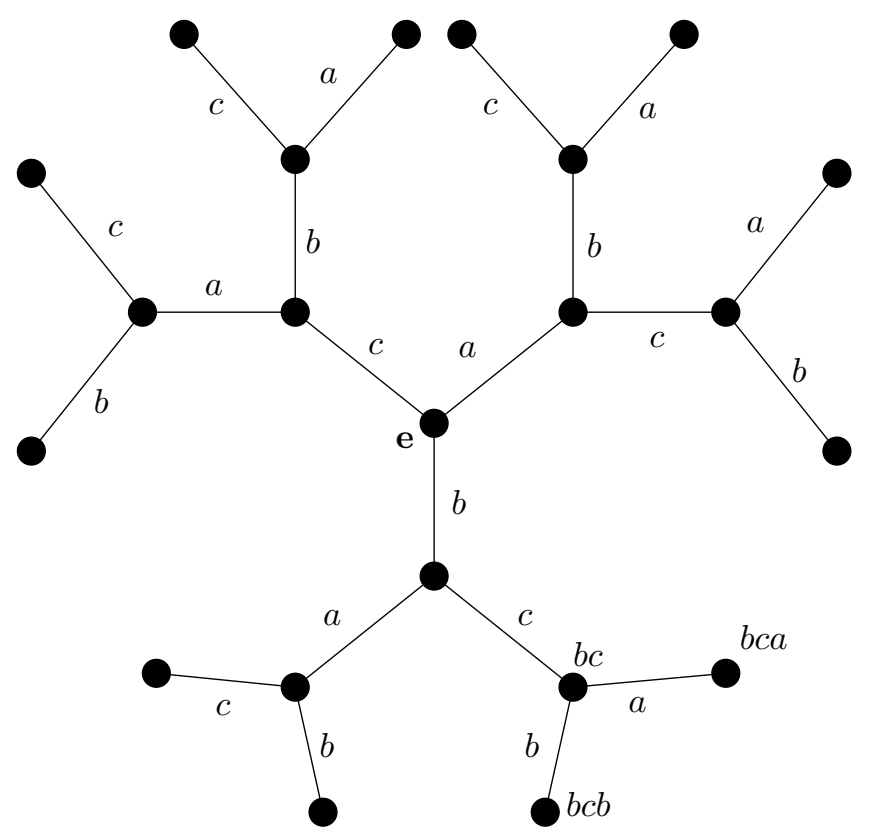

Figure 1 . The binary tree $\left(d=2, a_{1}:=a, a_{2}:=b, a_{3}:=c\right)$

\subsection{Branching random walk in random environment on a tree}

Consider a nonabelian group $\mathbb{T}_{d}$ generated by elements $a_{1}, \ldots, a_{d+1}$, with the rule $a_{i}^{2}=\mathbf{e}, i=1, \ldots, d+1$. With $S=\left\{a_{1}, \ldots, a_{d+1}\right\}$, the corresponding Cayley graph becomes the usual $d$-ary tree, see Figure 1.

We have the following result:

Theorem 3.5. The branching random walk in random environment on the $d$-ary tree is transient if and only if there exists a collection of positive numbers $\lambda_{1}, \ldots, \lambda_{d+1}$, such that for all $\omega \in \operatorname{supp} Q$

$$
\lambda_{i}^{-1} \mu_{a_{i}}^{\omega}+\sum_{j \neq i} \lambda_{j} \mu_{a_{j}}^{\omega} \leq 1 \quad \text { for all } i=1, \ldots, d+1 .
$$

Proof. First, we prove that the existence of $\lambda_{1}, \ldots, \lambda_{d+1}$ satisfying (3) implies transience. On $\mathbb{T}_{d}$, any positive multiplicative function is trivial, so we will use Theorem 3.3.

Clearly, any $x \in \mathbb{T}_{d}$ can be uniquely represented as $x=b_{1} \ldots b_{m}$, where $b_{i} \in\left\{a_{1}, \ldots, a_{d+1}\right\}$, and $b_{j} \neq b_{j+1}$ for $j=1, \ldots, m-1$. Define for $k=$ $1, \ldots, d+1$

$$
G_{k}=\left\{x=b_{1} \ldots b_{m}: b_{m}=a_{k}\right\}
$$


and let $A_{k}(x)$ be the number of occurrences of $a_{k}$ in that representation $x=b_{1} \ldots b_{m}$. For any $x \neq \mathbf{e}$, consider the function

$$
\psi(x)=\lambda_{1}^{A_{1}(x)} \ldots \lambda_{d+1}^{A_{d+1}(x)} .
$$

This function is almost multiplicative in the sense of Definition 3.2, with

$$
r_{k}\left(a_{j}\right)= \begin{cases}\lambda_{j}, & \text { for } j \neq k \\ \lambda_{j}^{-1}, & \text { for } j=k .\end{cases}
$$

Moreover, from (3) it is straightforward to obtain that (1) holds, and so the branching random walk is transient (observe that, due to Condition B, the situation $\lambda_{i} \leq 1$ for all $i=1, \ldots, d+1$, is not possible).

Now, let us prove that, if the branching random walk is transient, then there exist $\lambda_{1}, \ldots, \lambda_{d+1}$ satisfying (3).

First, let us observe that the main result of [12] (Theorem 2.1) is valid also for the model of the present paper (i.e., when the immediate descendants of a particle are not supposed to be independent). To see that this is true, one can reason as follows:

(i) One has to prove the modified version of Lemma 3.1 of [12], specifically, that transience implies that there exists a function $f: \mathcal{G} \rightarrow \mathbb{R}_{+}$ such that for all $x \in \mathcal{G} \backslash\{\mathbf{e}\}$

$$
\sum_{s \in S} \mu_{s}^{\omega_{x}} f(x s)=f(x)
$$

Similarly to [12], such a function can be constructed in the following way: turn e into an absorbing state, and let $f(x)$ be the expectation of the total number of particles absorbed in e. Moreover, it is clear that there exists a site $x$ (in fact, infinitely many of such sites) such that $f(x)<1$, because otherwise we would be able to construct a recurrent seed.

(ii) Then, note that the functions $h^{\xi}$ and $H_{i}$ (defined in formulas (2.1) and (2.2) of [12]) only depend on the transition and branching probabilities through the mean offsprings sent to the neighbouring sites (i.e., through the quantities we call $\mu_{s}^{\omega}$ ).

(iii) The rest of the proof of Theorem 2.1 of [12] goes through smoothly in this generalized situation as well.

Then, with this observation, we can apply Theorem 2.1 of [12] to the branching random walk in random environment on a tree (in the sense of [12]) with $d+1$ types of sites. The rule of choosing the type of the site is the following: a site of type $j$ has descendants of types $\{1, \ldots, d+1\} \backslash\{j\}$. (The root, in fact, should have descendants of types $1, \ldots, d+1$, but that does not change anything.) In the notation of [12], we obtain that, if the 
branching random walk is transient, then there exist $\lambda_{1}, \ldots, \lambda_{d+1}$ such that $\lambda_{i} \geq H_{i}\left(\lambda_{1}, \ldots, \lambda_{i-1}, \lambda_{i+1}, \ldots, \lambda_{d+1}\right)$, which is equivalent to $(3)$.

\subsection{Branching random walk in random environment on an integer lattice}

Here we suppose that $\mathcal{G}=\mathbb{Z}^{d}$, and so we write the group operation additively. The generating set $S$ is supposed to contain $\pm e_{i}, i=1, \ldots, d$, where $e_{i}$ are the coordinate vectors of $\mathbb{Z}^{d}$. Any positive multiplicative function on $\mathbb{Z}^{d}$ has the form $\varphi(y)=\lambda^{u \cdot y}, \lambda>0, u \in \mathbb{S}^{d-1}$, and $u \cdot y$ is the scalar product, where $\mathbb{S}^{d-1}$ is the unit sphere. So, the following result (cf. Theorem 1.6 of [4]) is a direct consequence of Corollary 3.4:

Theorem 3.6. Suppose that there exist $u \in \mathbb{S}^{d-1}, \lambda>0$ such that for all $\omega \in \operatorname{supp} Q$ we have

$$
\sum_{y \in S} \mu_{y}^{\omega} \lambda^{u \cdot y} \leq 1
$$

Then the branching random walk in random environment is transient.

As noted in [4], the existence of $u, \lambda$ satisfying (4) is also necessary for the transience in the case $d=1, S=\{-1,1\}$. In [13] it was proved that (4) is necessary and sufficient for the transience in the case when branching and transition probabilities are chosen independently. In fact, the author has good reasons to conjecture that the following holds:

Conjecture. If the branching random walk in random environment in $\mathbb{Z}^{d}$ is transient, then there exist $u \in \mathbb{S}^{d-1}, \lambda>0$ such that for all $\omega \in \operatorname{supp} Q$ we have

$$
\sum_{y \in S} \mu_{y}^{\omega} \lambda^{u \cdot y} \leq 1
$$

\section{References}

[1] S. Albeverio, L.V. Bogachev, S.A. Molchanov, E.B. Yarovaya, Annealed moment Lyapunov exponents for a branching random walk in a homogeneous random branching environment. Markov Process. Relat. Fields 6 (2000) (4), 473-516.

[2] J.-B. Baillon, P. Clément, A. Greven, F. den Hollander, A variational approach to branching random walk in random environment. Ann. Probab. 21 (1993), 290317.

[3] F. Comets, M.V. Menshikov, S.Yu. Popov, One-dimensional branching random walk in random environment: a classification. Markov Process. Relat. Fields 4 (1998) (4), 465-477.

[4] F. Comets, S. Popov, On multidimensional branching random walks in random environment. ArXiv math.PR/0507126. (2006) To appear in: Ann. Probab.

[5] A. Devulder, The speed of a branching system of random walks in random environment. (2006) Preprint. 
[6] J. Engländer, Branching Brownian motion with 'mild' Poissonian obstacles. (2005) Preprint, ArXiv math.PR/0508585.

[7] K. Fleischmann and A. Greven, Localization and selection in a mean field branching random walk in a random environment. Ann. Probab. 20 (1992) (4), 21412163.

[8] N. Gantert, S. Müller, The critical branching random walk is transient. ArXiv math.PR/0510556. (2005) To appear in: Markov Processes Relat. Fields.

[9] A. Greven, F. den Hollander, Branching random walk in random environment: phase transitions for local and global growth rates. Probab. Theory Related Fields 91 (1992), 195-249.

[10] F. den Hollander, M.V. Menshikov, S.Yu. Popov, A note on transience versus recurrence for a branching random walk in random environment. J. Statist. Phys. 95 (1999) (3-4), 587-614.

[11] F.P. Machado, S.Yu. Popov, One-dimensional branching random walk in a Markovian random environment. J. Appl. Probab. 37 (2000) (4), 1157-1163.

[12] F.P. Machado, S.Yu. Popov, Branching random walk in random environment on trees. Stochastic Process. Appl. 106 (2003) (1), 95-106.

[13] S. Müller, Recurrence and transience for branching random walks in an iid random environment. (2006) Preprint.

[14] P. Révész, Supercritical branching random walk in $d$-dimensional random environment. Applied Statistical Science, III, 41-51, Nova Sci. Publ., Commack, NY, 1998.

[15] S. Volkov, Branching random walk in random environment: fully quenched case. Markov Processes Relat. Fields 7 (2001) (2), 349-353. 